\title{
Excess Body Mass Index-Years, a Measure of Degree and Duration of Excess Weight, and Risk for Incident Diabetes
}

\author{
Joyce M. Lee, MD, MPH, Achamyeleh Gebremariam, MS, Sandeep Vijan, MD, and James G. \\ Gurney, PhD \\ Division of Pediatric Endocrinology (Dr Lee); Child Health Evaluation and Research Unit, Division \\ of General Pediatrics (Dr Lee and Mr Gebremariam); and Department of Internal Medicine (Dr \\ Vijan), University of Michigan, Ann Arbor; Veterans Affairs Health Services Research and \\ Development Service (Dr Vijan), Ann Arbor, Michigan; and Department of Epidemiology and \\ Cancer Control, St Jude Children's Research Hospital, Memphis, Tennessee (Dr Gurney)
}

\section{Abstract}

Objective-To evaluate the relation between excess body mass index (BMI)-years, a measure of the degree to which an individual's BMI (calculated as weight in kilograms divided by height in meters squared) exceeds the reference BMI and the duration for which he or she carries excess BMI, and incident diabetes.

Design-Longitudinal analysis.

Setting-United States of America.

Participants-A total of 8157 adolescents and young adults aged 14 to 21 years at the start of the National Longitudinal Survey of Youth 1979 with self-reported measures of height, weight, and diabetes status (type unspecified) from 1981 through 2006.

Main Exposure-Excess BMI-years, which were calculated by subtracting the reference BMI (25.0 for adults or 85th percentile for adolescents) from the actual BMI for each study year and cumulating excess BMI for the study duration.

Main Outcome Measure-We conducted logistic regression models to predict presumed type 2 diabetes (after excluding presumed type 1 diabetes) as a function of age, sex, race, excess BMIyears, and specific interactions.

Results-A higher level of excess BMI-years was associated with an increased risk of diabetes. For example, on average, white men aged 40 years with 200 excess BMI-years had 2.94 times (95\% confidence interval, 2.36-3.67) higher odds of developing diabetes compared with men of the same age and race with 100 excess BMI-years. For a given level of excess BMI-years, younger compared with older and Hispanic and black compared with white individuals had higher risk of

Correspondence: Joyce M. Lee, MD, MPH, Child Health Evaluation and Research Unit, University of Michigan, 300 North Ingalls Building, Room 6E18, Campus Box 5456, Ann Arbor, MI 48109-5456 (joyclee@umich.edu).

Author Contributions: Drs Lee and Gurney and Mr Gebremariam had full access to all the data in the study and take responsibility for the integrity of the data and the accuracy of the data analysis. Study concept and design: Lee, Vijan, and Gurney. Acquisition of data: Lee and Gebremariam. Analysis and interpretation of data: Lee, Gebremariam, Vijan, and Gurney. Drafting of the manuscript: Lee. Critical revision of the manuscript for important intellectual content: Lee, Gebremariam, Vijan, and Gurney. Statistical analysis: Lee, Gebremariam, Vijan, and Gurney. Administrative, technical, and material support: Lee and Gebremariam. Study supervision: Vijan and Gurney.

Financial Disclosure: None reported.

Additional Information: This work used the Biostatistics Core(s) of the Michigan Diabetes Research and Training Center. 
developing diabetes. Our study is limited by use of self-reported data without specification of diabetes type.

Conclusions-Because younger compared with older individuals have a higher risk of selfreported diabetes for a given level of excess BMI-years and cumulative exposure to excess BMI is increasing among younger US birth cohorts, public health interventions should target younger adults.

The link between obesity and type 2 diabetes (T2D) has been well characterized by epidemiologic studies. ${ }^{1-12}$ The majority of studies have focused on the degree of obesity as an important risk factor, with a higher absolute body mass index (BMI; calculated as weight in kilograms divided by height in meters squared) conferring a greater risk of incident T2D. Less studied is the effect of obesity duration as an independent risk factor for T2D incidence. At least 6 studies ${ }^{9,13-17}$ have reported that, independent of absolute BMI, longer obesity duration is associated with higher rates of T2D. However, these studies were based on populations that are unique (e.g., Pima Indians, ${ }^{13}$ non-US-dwelling individuals ${ }^{14-16}$ ) or racially homogeneous, ${ }^{9,17}$ which may not be generalizable to the broad US population. Furthermore, they used relatively crude measures of duration by comparing individuals with obesity duration of less than 10 years with those with duration of greater than or equal to10years, which does not account for degree of obesity as a potentially important risk factor. $^{13}$

Understanding the impact of both degree and duration of obesity on incident T2D is critical, given the childhood obesity epidemic. The tripling of obesity rates for US children during the last 30 to 40 years ${ }^{18}$ implies that younger generations of individuals are carrying a longer duration of obesity over their lifetime. ${ }^{19}$ Furthermore, documented increases in morbid obesity (BMI percentile 299 th) among US children ${ }^{20}$ mean that the overall degree of obesity is increasing. This increasing degree and duration of obesity for younger generations may have important consequences for future obesity-related morbidity and mortality rates.

We therefore sought to evaluate the relation between degree and duration of excess BMI and incident diabetes through analysis of a term analogous to smoking pack-years, which we call excess BMI-years, a composite measure of the degree to which an individual's BMI exceeds his or her reference BMI and the duration for which he or she carries excess BMI. Although use of the cumulative smoking analogy applied to excess BMI was first conceptualized by Brancati et al, ${ }^{12}$ no studies have attempted to characterize such a term and its relation with incident diabetes, to our knowledge. Understanding this relation is important for increasing the reliability of US diabetes projections and to better understand the consequences of increasing weight among younger US generations.

\section{METHODS}

The National Longitudinal Survey of Youth 1979 (NLSY79) is a nationally representative, longitudinal cohort study. ${ }^{21}$ Participants, aged 14 to 21 years at the start, provided repeated measures of self-reported height and weight and self-reported onset of diabetes of unspecified type. The cohort retention rate was $80.9 \%$ by 2006 .

\section{MAIN EXPOSURE}

Weight was available for the following calendar years: 1981 to 1982,1985 to 1986,1988 to 1990, 1992 to 1993, and biyearly from 1994 to 2006; height was not available at every survey date, so the most recent height was used for BMI calculations. To calculate degree of excess BMI, we subtracted the reference BMI (defined as BMI thresholds for overweight of 25.0 for adults and 85th percentile [age- and sex-adjusted] for adolescents) from the actual BMI. For example, 23.4 corresponds to the 85 th percentile for a boy aged 15 years; the 
degree of obesity for a BMI of 25.0 would be $25.0-23.4=1.6$. Individuals could have a negative excess BMI if the actual was less than the reference BMI.

We accounted for duration of obesity by summing excess BMI over all previous years to calculate excess BMI-years (Table 1). Therefore, excess BMI values of -0.3 and 0.8 in 1981 and 1982 represent excess BMI-year values of -0.3 and $0.5(-0.3+0.8)$, respectively.

Because the NLSY79 was not conducted in consecutive years, we averaged the excess BMI between adjacent surveys to create a value for the missing year; i.e., the excess BMI value for 1987 was calculated by averaging values for 1986 and 1988 (Table 1). For example, an adult with a BMI of 30 for 10 years would accumulate 5 BMI-years for each of those years (5X $10=50$ excess BMI-years).

\section{MAIN OUTCOME MEASURE}

Incident diabetes was classified by an affirmative response to the question, "Has a doctor ever told you that you have diabetes or high blood sugar?" Although participants indicated what month and year their diabetes was first diagnosed, no information was provided regarding diabetes type and no medical verification of the diagnosis was available. Type 1 diabetes of autoimmune origin, although rare, also can occur during adulthood in individuals who are usually of normal weight. We therefore excluded individuals who were of normal weight (BMI of <25) at the survey year just prior to their diagnosis. Additional definitions of T2D, as well as different reference BMI-years cutoffs of 22 and 30, were used in sensitivity analyses, as discussed herein.

\section{STUDY POPULATION}

Of 12686 respondents in the NLSY79, our sample consisted of the 8446 who answered the question regarding diabetes status in 2006. Individuals in the sample, compared with those not included, essentially were the same age (mean [SE], 19.74 [0.03]) vs 19.70 [0.06] years, respectively; $P=.56$ ) and had slightly higher BMI (mean [SE], 22.61 [0.05] vs 22.24 [0.08], respectively; $P<.001)$; a greater proportion of female (50.7\% vs $43.5 \%, P=.001)$ and nonwhite (6.2\% vs $5.2 \%$ for Hispanics, $14.4 \%$ vs $9.3 \%$ for non-Hispanic blacks, $P<.001$ ) individuals were included. Of 451 individuals diagnosed with diabetes, we excluded those who did not give a diagnosis date $(\mathrm{n}=6)$ or who reported onset of diabetes prior to the start of the survey $(n=43)$. After excluding 65 individuals with presumed type 1 diabetes based on their BMI prior to the date of diagnosis, a sample of 337 individuals with presumed T2D remained. Of the 7995 individuals who did not report having diabetes, we excluded those who were nonrespondents in $1981(\mathrm{n}=175)$, leaving a sample of 8157 individuals.

\section{STATISTICAL ANALYSIS}

We organized the data into a person-period data set. If an individual developed diabetes, BMI measurements subsequent to the diagnosis were not considered. Because each respondent was contributing multiple observations, we accounted for repeated measures using generalized estimating equations and conducted logistic regression models to predict incident diabetes as a function of age, sex, race, and excess BMI. We tested the 2-way interactions of sex $\mathrm{X}$ race, sex $\mathrm{X}$ age, sex $\mathrm{X}$ excess BMI-years, race $\mathrm{X}$ age, race $\mathrm{X}$ excess BMI-years, and age $\mathrm{X}$ excess BMI years. We then refined the final model to include significant (race $\mathrm{X}$ excess BMI-years and age $\mathrm{X}$ excess BMI-years) or marginally significant (race $\mathrm{X}$ age $[P=.08]$ and sex $\mathrm{X}$ excess BMI-years[ $P=.06]$ ) interactions. To display the effect of interactions, we created lowess smoothed graphs of the predicted incidence of diabetes as a function of age and excess BMI-years, as well as of age, race, and excess BMI-years. We also created receiver operating characteristic curves and calculated the area under the curve 
(AUC) to compare the predictive capacity of the model containing excess BMI-years vs a cross-sectional model of baseline BMI for the same cohort.

Many studies have shown a relation between socioeconomic status and risk of diabetes. 22-24 When we included baseline family income (dichotomized as the highest 3 quartiles compared with the lowest quartile) as a covariate, no significant association of socioeconomic status with risk of diabetes was observed, and its inclusion in the model did not change our findings (data not shown). Therefore, given $20.8 \%$ missing data for income and the lack of evidence for confounding, we elected not to include income in our final model. Also, we did not include smoking status in the model because it was missing for most survey years.

To account for missing BMI values (7.8\% of all measurements), we used race, sex, age, and all available BMI data to create 5 multiple imputation data sets using imputation by chained equations. We performed analyses on each of the multiple imputation data sets and combined results across the data sets using Stata software, version 10.0 (StataCorp LP, College Station, Texas). Because of the longitudinal nature of our analyses, we did not use the unique survey weights provided by the NLSY79 for each survey year except for characterizing the baseline study population. This study was classified as exempt by the University of Michigan Institutional Review Board.

\section{RESULTS}

Table 2 shows demographic and weight characteristics at baseline and by 2006.

Approximately $4 \%$ of the cohort was obese, an estimate consistent with national data when the study was implemented and prior to the dramatic increase in obesity in the 1980s. More than half were female, with ample representation of nonwhite individuals. After 25 years of follow-up, the mean age for the population was 44.9 years; 337 individuals had developed diabetes (mean age at onset, 37.2 years). Rates of obesity and diabetes were higher for nonwhite compared with white individuals.

For the overall population, mean excess BMI-years remained close to $0(-1.0)$ averaged across ages. However, mean excess BMI-years increased with age: $-10.2,-7.7,1.1$, and 11.9 at ages $25,30,35$, and 40 years, respectively. Figure 1 shows a smoothed histogram of the distribution of excess BMI-years at specific ages for the overall and the obese population.

We found that higher excess BMI-years are associated with a greater risk of self-reported incident diabetes. For example, on average, white men aged 40 years with 200 cumulative excess BMI-years have 2.94 times (95\% CI, 2.36-3.67) higher odds of developing diabetes compared with men of the same age and race with 100 excess BMI-years. Interactions for race $\mathrm{X}$ excess BMI-years $(P=.01)$ and age $\mathrm{X}$ excess BMI-years $(P=.001)$ were apparent, as illustrated in Figure 2 and Figure 3.

Figure 2 displays a lowess graph showing the predicted incidence of diabetes at a specific age as a function of excess BMI-years, given that diabetes had not occurred at a previous age. For example, the green line represents the predicted incidence of diabetes at age 25 years as a function of excess BMI-years. This line is truncated because these individuals haven't yet had the opportunity to develop higher levels of excess BMI-years. The orange line represents the predicted incidence of developing diabetes at age 30 years, conditional on not having developed diabetes by age 29 years. Analogously, the blue and red lines represent the predicted incidence of developing diabetes at ages 35 and 40 years, conditional on not having developed diabetes by ages 34 and 39 years, respectively. At an excess BMI- 
years value of 200, the predicted incidence of developing diabetes was higher for youngeronset (age 30 years) compared with olderonset (ages 35 and 40 years) diabetes.

Figure 3 displays similar lowess graphs stratified by race. In Table 3 we present tests of interactions between race and excess BMI-years on diabetes risk according to specific ages and levels of excess BMI-years. For example, for levels of excess BMI-years ranging from 0 to 200, Hispanic and black compared with white individuals and Hispanic compared with black individuals had higher odds of developing diabetes across the various ages. Racial differences in risk of diabetes were not significant at higher (300-500) levels of excess BMIyears.

Finally, the receiver operating characteristic curve for the model containing excess BMIyears (AUC, 0.85 [95\% confidence interval, 0.83-0.87]) had a 4.6\% higher AUC than did the cross-sectional model substituting excess BMI-years with baseline BMI $(0.80$ [0.78-0.82] $(P<.001)$.

\section{COMMENT}

This analysis demonstrates and quantifies the effect of excess BMI-years, a measure of the degree and duration of overweight during young and middle adulthood, on the risk for development of diabetes in adulthood. We show that higher excess BMI-years are associated with an increased risk of diabetes and that this relation differs by age. Excess BMI-years for excess BMI-year, younger compared with older individuals have a higher risk of developing diabetes.

One hypothesis to account for our findings is that a given amount of excess BMI when carried earlier in the life course may be more diabetogenic than the same amount of weight carried later in the life course. We recently documented 19 the extent to which younger US birth cohorts are accumulating excess weight much earlier in the life course, achieving rates of obesity by adolescence or young adulthood that previously only had been reached at midadulthood by older birth cohorts. Our findings suggest that these trends may result in steeper increases in diabetes for younger compared with older adults. Further studies are needed to confirm these findings, but if excess BMI earlier rather than later in the life course is associated with a higher risk of diabetes, this would suggest that weight interventions for diabetes prevention may be more effective at younger compared with older ages and that public health programs may need to prioritize accordingly.

We are unaware of longitudinal studies that have documented a differential probability of developing diabetes within specific age groups for a given degree of excess BMI. However, we note that our findings are consistent with those of a study ${ }^{25}$ of approximately 2400 adults from a managed care plan in Portland, Oregon, with new-onset T2D diagnosed between 1996 and 1998 and with BMI values available within 3 months of their diagnosis; the findings showed that individuals with a younger age at onset had a higher mean BMI compared with individuals with an older age at onset. However, the data for that study were cross-sectional and did not assess prior history of excess BMI.

One study ${ }^{26}$ evaluated age interactions between weight gain and fasting plasma glucose levels among a population of men from a Veterans Administration Aging Study in Boston, Massachusetts. It followed up approximately 1400 men between the ages of 21 and 81 years for at least 12 years. Of interest, weight gain at older ages compared with younger ages had a greater adverse effect on fasting plasma glucose levels, which contrasts with our findings. However, this study did not use diabetes as the outcome measure, and its population was substantially older. Furthermore, because it only consisted of men who were armed-forces veterans, the generalizability of the study findings is limited. 
Alternatively, our findings may be reflective of the pathophysiologic development of T2D during the life course. ${ }^{27,28} \mathrm{~A}$ combination of insulin resistance and $\beta$-cell failure is required for developing diabetes, but the relative influence of these factors may differ at younger compared with older ages. Failure of $\beta$-cells may represent a more important determinant for individuals diagnosed with diabetes at older ages, given a number of studies ${ }^{29-31}$ that have shown that $\beta$-cell function declines with age. In contrast, insulin resistance, which is largely determined by obesity, may represent a more important determinant for individuals diagnosed with diabetes at younger ages.

Our findings regarding possible racial differences in the relation of excess BMI-years with diabetes at lower levels of excess BMI-years are consistent with those of studies ${ }^{32,33}$ reporting differential risk of diabetes across racial subgroups at specific BMI ranges. For example, a nationally representative study ${ }^{33}$ reported that at lower levels of BMI (22-31), blacks had a higher risk of diabetes compared with whites but at higher levels ( 232 ), no statistical differences were observed. Racial disparities in diabetes prevalence already exist in the adult population. ${ }^{34}$ This trend will only be magnified by the disproportionate number of US nonwhite adolescents and young adults who are overweight compared with their white counterparts ${ }^{35}$ and the additional risk of diabetes for a given level of excess BMIyears for nonwhite individuals.

Strengths of our study include the fact that the NLSY79 was nationally representative, racially diverse, followed up individuals for a long duration starting in adolescence, had available multiple measures of BMI, and had a high retention rate. We also showed that our model containing excess BMI-years was more predictive than a model using BMI measured at a single time point.

We acknowledge limitations of our study. The NLSY79 used self-reported weight and height. Because individuals tend to underreport their weight and overreport their height, ${ }^{36}$ this potential misclassification may lead to an underestimation of the associations we found. A small proportion of the population consisted of adolescents aged 14 to 17 in 1981; studies have shown that self-reported height and weight provide accuracy of $96 \%$ in classification of weight status in teenagers. However, because we used the last reported height to calculate BMI, this may have resulted in an overestimation of BMI for those who had not completed their linear growth.

The NLSY79 also used self-reported diabetes without specification of type of diabetes. Although it is unlikely that an individual would not know that he or she had type 1 diabetes, undiagnosed T2D is possible. We are unaware of studies that have compared rates of undiagnosed diabetes according to age. Screening guidelines traditionally have focused on older adults (aged $\geq 45$ years) ${ }^{37,38}$; therefore, one might speculate that undiagnosed T2D could be higher among younger compared with older adults. If this were true, we would expect that the association of increased diabetes with excess BMI-years would be underestimated in our study.

Although diabetes type was not specified, we ran sensitivity analyses using 2 additional definitions of T2D. We eliminated individuals who were of normal weight $(\mathrm{BMI}$ of $<25.0)$ during the survey year just prior to their diagnosis and who were 30 years or younger at the time of diagnosis $(\mathrm{n}=30)$. We also included all individuals with diagnosed diabetes regardless of weight status or age. For these sensitivity analyses, the age X excess BMIyears interaction remained significant, although the race $\mathrm{X}$ excess BMI-years interaction was not. We also evaluated the reference BMI at levels of 22.0 and 30.0 in sensitivity analyses, finding no appreciable differences in model coefficients or AUC (data not shown). 
The definition of diabetes changed in 1997, with a lowering of the fasting plasma glucose threshold from 140 to $126 \mathrm{mg} / \mathrm{dL}$; therefore, it is likely that diabetes case ascertainment increased over time. Because the youngest individual in the cohort was aged 14 years, we do not have weight information from early childhood; therefore, we could not account for excess BMI-years from birth. Finally, because of the age of the cohort, we do not have information regarding diabetes risk in late adulthood.

In conclusion, excess BMI-years were associated with self-reported incident diabetes, and this risk was higher for younger adults and possibly for black and Hispanic adults compared with white adults at a given level of excess BMI-years. This information is critical for increasing the reliability of predictions of diabetes rates and diabetes burden and for achieving a better understanding of the consequences of increasing weight among younger generations in the United States. Future public health interventions focused on diabetes prevention may need to target younger nonwhite individuals to prevent a further acceleration in diabetes rates.

\section{Acknowledgments}

Funding/Support: Dr Lee was supported by grant K08DK082386 and the work by grant OK020572 from the National Institute of Diabetes and Digestive and Kidney Diseases and the Clinical Sciences Scholars Program at the University of Michigan.

Role of the Sponsor: The funding sources had no role in the study design; the collection, analysis, and interpretation of data; the writing of the article; or the decision to submit the article for publication.

\section{References}

1. Colditz GA, Willett WC, Rotnitzky A, Manson JE. Weight gain as a risk factor for clinical diabetes mellitus in women. Ann Intern Med. 1995; 122(7):481-486. [PubMed: 7872581]

2. Colditz GA, Willett WC, Stampfer MJ, et al. Weight as a risk factor for clinical diabetes in women. Am J Epidemiol. 1990; 132(3):501-513. [PubMed: 2389754]

3. Skarfors ET, Selinus KI, Lithell HO. Risk factors for developing non-insulin dependent diabetes: a 10 year follow up of men in Uppsala. BMJ. 1991; 303(6805):755-760. [PubMed: 1932936]

4. Wilson PW, McGee DL, Kannel WB. Obesity, very low density lipoproteins, and glucose intolerance over fourteen years: the Framingham Study. Am J Epidemiol. 1981; 114(5):697-704. [PubMed: 7304597]

5. Feskens EJ, Kromhout D. Cardiovascular risk factors and the 25-year incidence of diabetes mellitus in middle-aged men: the Zutphen Study. Am J Epidemiol. 1989; 130(6):1101-1108. [PubMed: 2589303]

6. Medalie JH, Papier CM, Goldbourt U, Herman JB. Major factors in the development of diabetes mellitus in 10,000 men. Arch Intern Med. 1975; 135(6):811-817. [PubMed: 1130926]

7. Jarrett RJ, Keen H, McCartney P. The Whitehall Study: ten year follow-up report on men with impaired glucose tolerance with reference to worsening to diabetes and predictors of death. Diabet Med. 1984; 1(4):279-283. [PubMed: 6242817]

8. Ohlson LO, Larsson B, Svärdsudd K, et al. The influence of body fat distribution on the incidence of diabetes mellitus: 13.5 years of follow-up of the participants in the study of men born in 1913 . Diabetes. 1985; 34(10):1055-1058. [PubMed: 4043554]

9. Holbrook TL, Barrett-Connor E, Wingard DL. The association of lifetime weight and weight control patterns with diabetes among men and women in an adult community. Int J Obes. 1989; 13(5):723729. [PubMed: 2583926]

10. Manson JE, Nathan DM, Krolewski AS, Stampfer MJ, Willett WC, Hennekens CH. A prospective study of exercise and incidence of diabetes among US male physicians. JAMA. 1992; 268(1):6367. [PubMed: 1608115]

11. Hanson RL, Narayan KM, McCance DR, et al. Rate of weight gain, weight fluctuation, and incidence of NIDDM. Diabetes. 1995; 44(3):261-266. [PubMed: 7883111] 
12. Brancati FL, Wang N-Y, Mead LA, Liang K-Y, Klag MJ. Body weight patterns from 20 to 49 years of age and subsequent risk for diabetes mellitus: the Johns Hopkins Precursors Study. Arch Intern Med. 1999; 159(9):957-963. [PubMed: 10326937]

13. Everhart JE, Pettitt DJ, Bennett PH, Knowler WC. Duration of obesity increases the incidence of NIDDM. Diabetes. 1992; 41(2):235-240. [PubMed: 1733815]

14. Carlsson S, Persson P-G, Alvarsson M, et al. Weight history, glucose intolerance, and insulin levels in middle-aged Swedish men. Am J Epidemiol. 1998; 148(6):539-545. [PubMed: 9753008]

15. Modan M, Karasik A, Halkin H, et al. Effect of past and concurrent body mass index on prevalence of glucose intolerance and type 2 (non-insulin-dependent) diabetes and on insulin response: the Israel Study of glucose Intolerance, Obesity and Hypertension. Diabetologia. 1986; 29(2):82-89. [PubMed: 3516770]

16. Sakurai Y, Teruya K, Shimada N, et al. Association between duration of obesity and risk of noninsulin-dependent diabetes mellitus: the Sotetsu Study. Am J Epidemiol. 1999; 149(3):256-260. [PubMed: 9927221]

17. Abdullah A, Stoelwinder J, Shortreed S, et al. The duration of obesity and the risk of type 2 diabetes. Public Health Nutr. 2011; 14(1):119-126. [PubMed: 20587115]

18. Ogden CL, Flegal KM, Carroll MD, Johnson CL. Prevalence and trends in overweight among US children and adolescents, 1999-2000. JAMA. 2002; 288(14):1728-1732. [PubMed: 12365956]

19. Lee JM, Pilli S, Gebremariam A, et al. Getting heavier, younger: trajectories of obesity over the life course. Int J Obes (Lond). 2010; 34(4):614-623. [PubMed: 19949415]

20. Skelton JA, Cook SR, Auinger P, Klein JD, Barlow SE. Prevalence and trends of severe obesity among US children and adolescents. Acad Pediatr. 2009; 9(5):322-329. [PubMed: 19560993]

21. United States Department of Labor, Bureau of Labor Statistics. [February 4, 2007] National Longitudinal Surveys. February 4, 2007. http://www.bls.gov/nls/

22. Robbins JM, Vaccarino V, Zhang H, Kasl SV. Excess type 2 diabetes in African-American women and men aged 40-74 and socioeconomic status: evidence from the Third National Health and Nutrition Examination Survey. J Epidemiol Community Health. 2000; 54(11):839-845. [PubMed: 11027198]

23. Robbins JM, Vaccarino V, Zhang H, Kasl SV. Socioeconomic status and diagnosed diabetes incidence. Diabetes Res Clin Pract. 2005; 68(3):230-236. [PubMed: 15936465]

24. Brancati FL, Whelton PK, Kuller LH, Klag MJ. Diabetes mellitus, race, and socioeconomic status: a population-based study. Ann Epidemiol. 1996; 6(1):67-73. [PubMed: 8680628]

25. Hillier TA, Pedula KL. Characteristics of an adult population with newly diagnosed type 2 diabetes: the relation of obesity and age of onset. Diabetes Care. 2001; 24(9):1522-1527. [PubMed: 11522693]

26. Borkan GA, Sparrow D, Wisniewski C, Vokonas PS. Body weight and coronary disease risk: patterns of risk factor change associated with long-term weight change: the Normative Aging Study. Am J Epidemiol. 1986; 124(3):410-419. [PubMed: 3740041]

27. Kahn SE. Clinical review 135: the importance of $\beta$-cell failure in the development and progression of type 2 diabetes. J Clin Endocrinol Metab. 2001; 86(9):4047-4058. [PubMed: 11549624]

28. Kahn SE, Zraika S, Utzschneider KM, Hull RL. The beta cell lesion in type 2 diabetes: there has to be a primary functional abnormality. Diabetologia. 2009; 52(6):1003-1012. [PubMed: 19326096]

29. Chiu KC, Lee NP, Cohan P, Chuang L-M. Beta cell function declines with age in glucose tolerant Caucasians. Clin Endocrinol (Oxf). 2000; 53(5):569-575. [PubMed: 11106917]

30. Chang AM, Smith MJ, Galecki AT, Bloem CJ, Halter JB. Impaired $\beta$-cell function in human aging: response to nicotinic acid-induced insulin resistance. J Clin Endocrinol Metab. 2006; 91(9): 3303-3309. [PubMed: 16757523]

31. Gumbiner B, Polonsky KS, Beltz WF, Wallace P, Brechtel G, Fink RI. Effects of aging on insulin secretion. Diabetes. 1989; 38(12):1549-1556. [PubMed: 2684711]

32. Lipton RB, Liao Y, Cao G, Cooper RS, McGee D. Determinants of incident noninsulin-dependent diabetes mellitus among blacks and whites in a national sample: the NHANES I Epidemiologic Follow-up Study. Am J Epidemiol. 1993; 138(10):826-839. [PubMed: 8237971]

33. Resnick HE, Valsania P, Halter JB, Lin X. Differential effects of BMI on diabetes risk among black and white Americans. Diabetes Care. 1998; 21(11):1828-1835. [PubMed: 9802729] 
34. Cowie CC, Rust KF, Ford ES, et al. Full accounting of diabetes and pre-diabetes in the U.S. population in 1988-1994 and 2005-2006. Diabetes Care. 2009; 32(2):287-294. [PubMed: 19017771]

35. Ogden CL, Carroll MD, Curtin LR, Lamb MM, Flegal KM. Prevalence of high body mass index in US children and adolescents, 2007-2008. JAMA. 2010; 303(3):242-249. [PubMed: 20071470]

36. Palta M, Prineas RJ, Berman R, Hannan P. Comparison of self-reported and measured height and weight. Am J Epidemiol. 1982; 115(2):223-230. [PubMed: 7058781]

37. US Preventive Services Task Force. Screening for type 2 diabetes mellitus in adults: US Preventive Services Task Force recommendation statement. Ann Intern Med. 2008; 148(11):846-854. published correction appears in Ann Intern Med. 2008;149(2):147. [PubMed: 18519930]

38. American Diabetes Association. Screening for type 2 diabetes. Diabetes Care. 2004; 27(suppl 1):S11-S14. [PubMed: 14693922] 


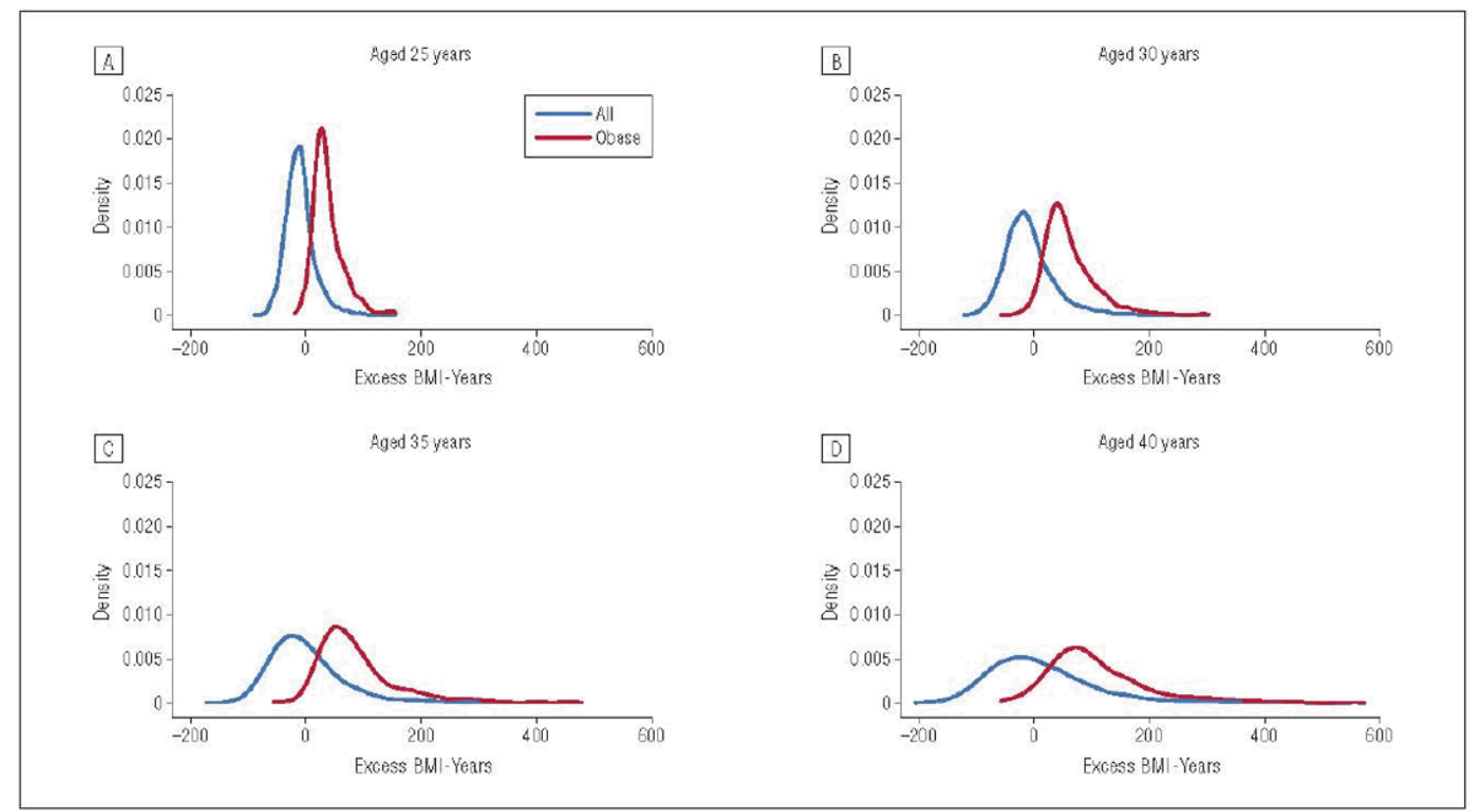

Figure 1.

Histograms of excess body mass index (BMI)-years for the overall cohort and for obese individuals (BMI 230.0 ) at 25 (A), 30 (B), 35 (C), and 40 (D) years. Imputed values for $\mathrm{BMI}$ are included in the figure. 


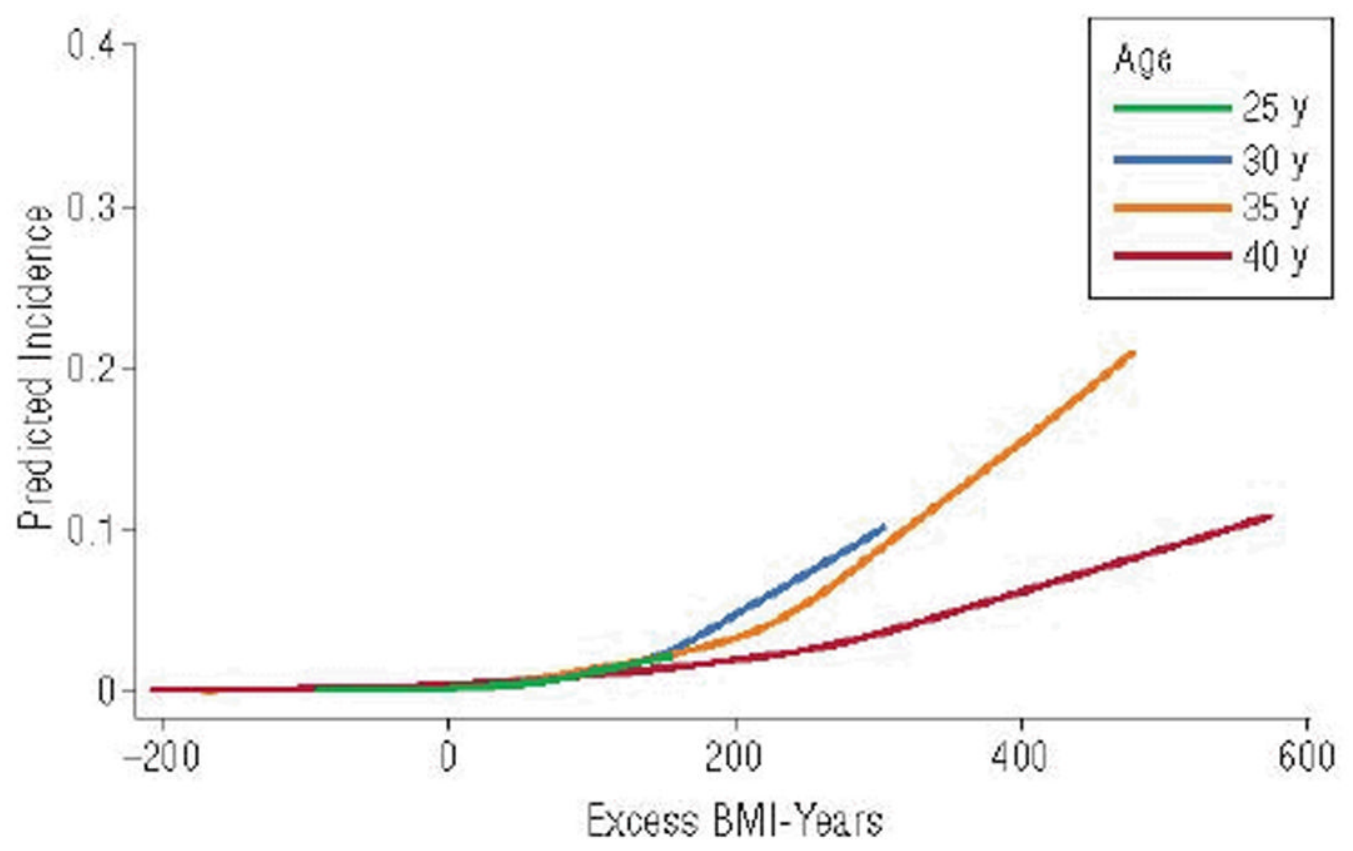

Figure 2.

Lowess graph showing the predicted first incidence of diabetes at a specific age, given that diabetes has not occurred at a previous age. For example, the blue line represents the predicted incidence of diabetes at age 35 years as a function of excess body mass index (BMI)-years, conditional on not having developed diabetes by age 34 years. Imputed values for BMI are included in the figure. 


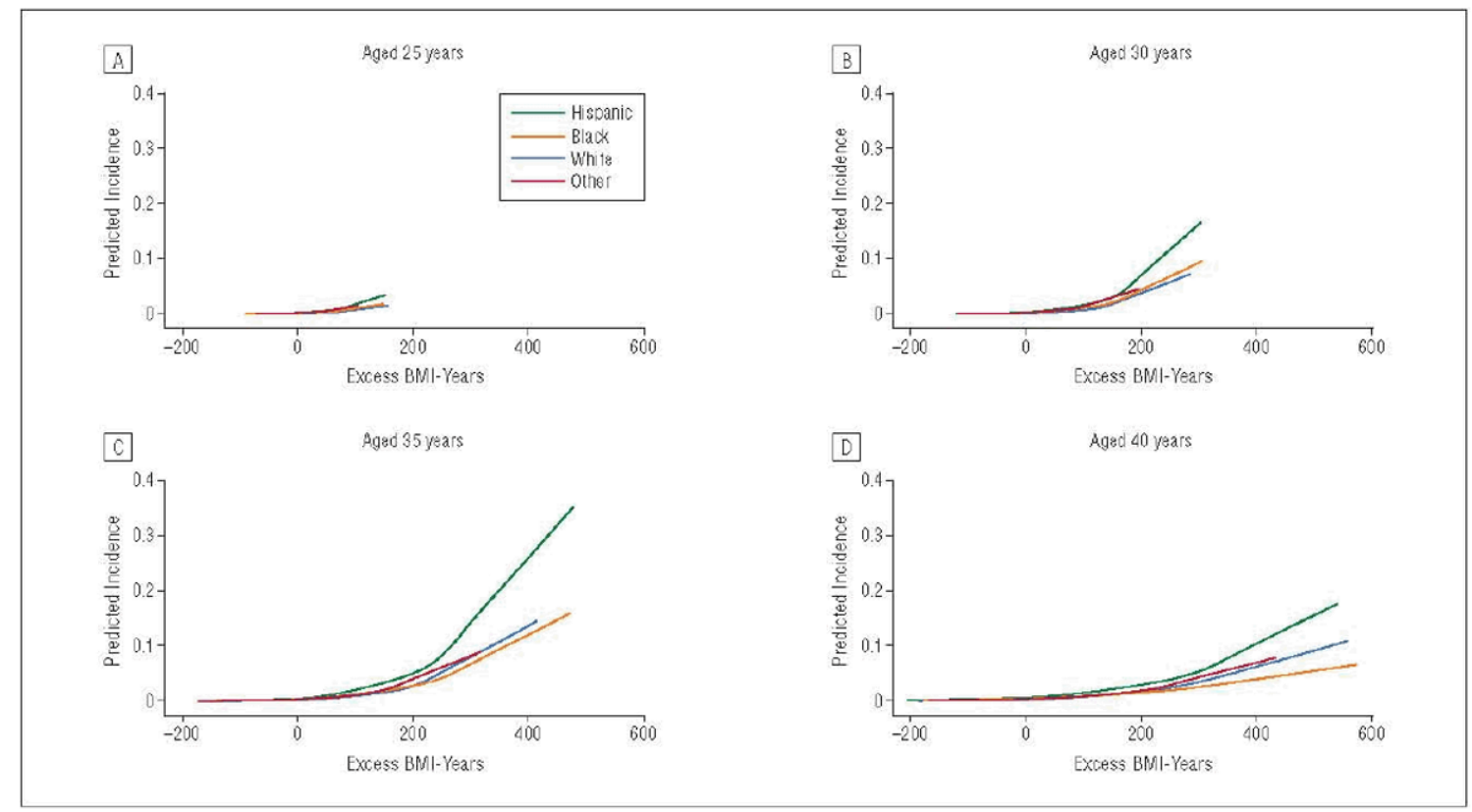

Figure 3.

Lowess graphs showing the predicted first incidence of diabetes at a specific age, given that diabetes has not occurred at a previous age, in relation to excess body mass index (BMI)years for individuals of non-Hispanic white, non-Hispanic black, Hispanic, and other race. 


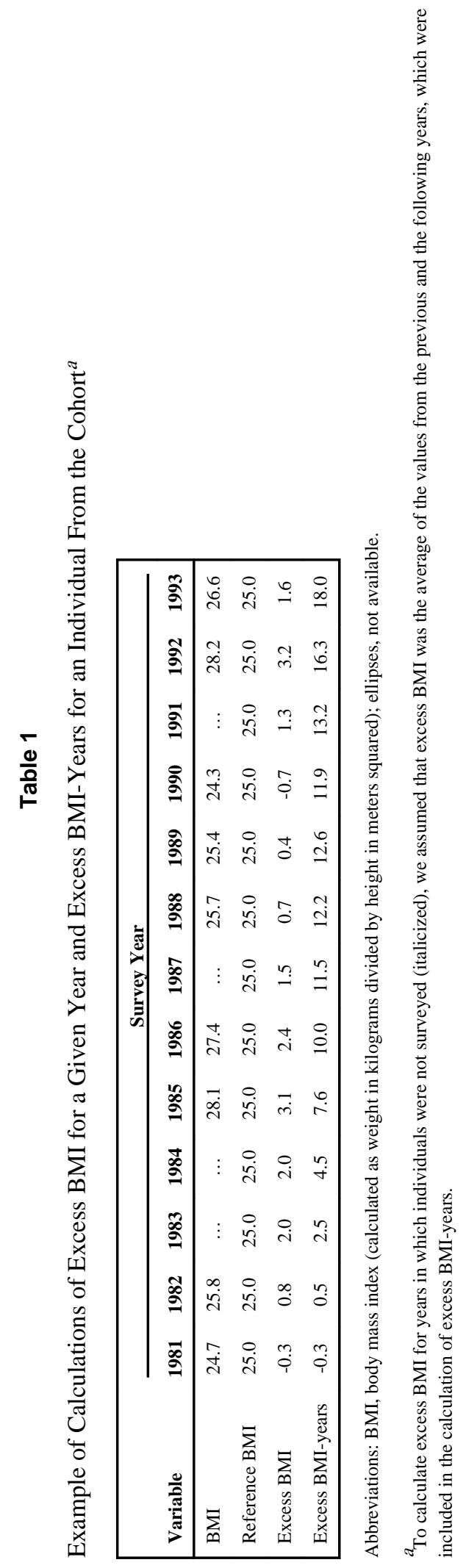

Arch Pediatr Adolesc Med. Author manuscript; available in PMC 2013 January 01. 


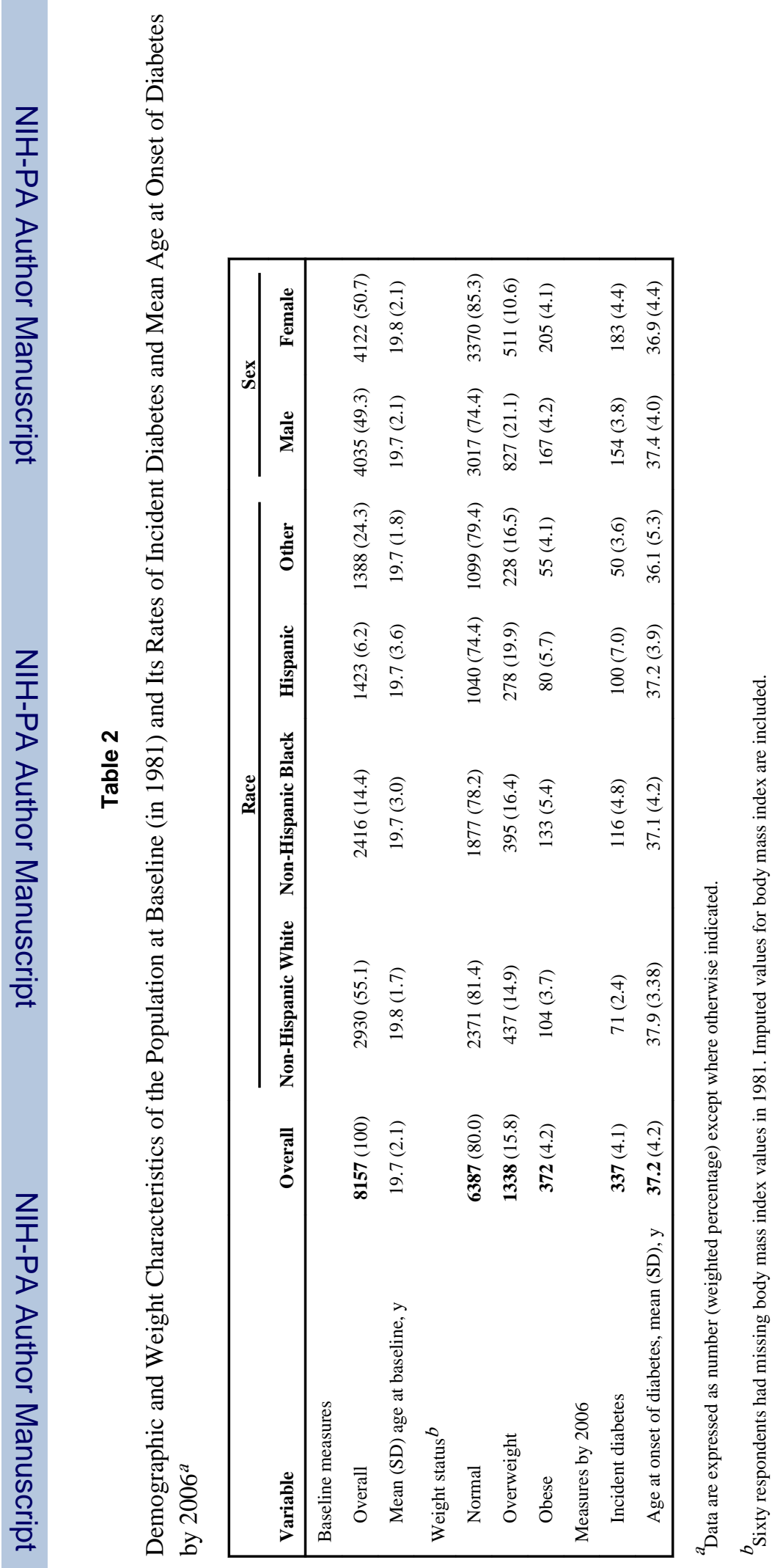


